\title{
STABLE BUNDLES ON PROJECTIVE CURVES: THEIR FILTRATIONS AND THEIR SUBBUNDLES
}

\author{
E. BALLICO
}

Let $X$ be a smooth genus $g$ projective curve. Here we study the maximal degree subbundles of the stable vector bundles on $X$ and the existence of stable vector bundles on $X$ with suitable filtrations by subbundles. Tools: Elementary transformations of vector bundles and pull-backs of bundles from lower genus curves.

\section{Introduction.}

Let $X$ be a smooth, projective curve of genus $g$. Let $F$ be a vector bundle on $X$ such that $F$ has an increasing filtration $\left\{F_{j}\right\}_{0 \leq j \leq k}$ by saturated subbundles with $F_{0}:=\{0\}$ and $F_{k}:=F$; set $a_{i}:=\operatorname{deg}\left(F_{i} / F_{i-1}\right)$ and $r_{i}:=\operatorname{rank}\left(F_{i} / F_{i-1}\right)$, $1 \leq i \leq k$; assume $F_{i} \neq F_{i-1}$ for every $i$, i.e. $r_{i}>0$ for every $i$; the ordered set of integers $\left(k ; a_{1}, r_{1}, \ldots, a_{k}, r_{k}\right)$ will be called the type (or the numerical type) of the filtration $\left\{F_{j}\right\}_{0 \leq j \leq k}$. If $g \geq 2$ we are interested in the existence of a stable vector bundle on $X$ with a filtration of type $\left(k ; a_{1}, r_{1}, \ldots, a_{k}, r_{k}\right)$. If $k=2$ a necessary condition is the inequality $a_{1} / r_{1}<a_{2} / r_{2}$. In [L2] H. Lange conjectured that this condition is also a sufficient condition. Recently, several cases of Lange's conjecture were solved by L. Brambila-Paz and H. Lange ([BL]) and by M. Teixidor i Bigas $([\mathbf{T}])$. We always assume characteristic 0 . We list here some of our main results (all proven in Section 3); for other results, see 3.1 and Section 4.

For an integer $a$, set $a_{+}=a_{-}=a$ if $\mathrm{a}$ is even and $a_{+}=a_{-}+2=a+1$ if a is odd.

Theorem 0.1. Fix integers $g, k, q, a_{i}, 1 \leq i \leq k, r_{i}, 1 \leq i \leq k$. Assume $r_{i}>0$ for every $i \geq 1, q \geq 1, k \geq 2,2 g+2>k+4 q$ if $q \geq 2, g \geq k$ if $q=1$. If $q=1$ assume $\left(a_{i+}+2\right) / r_{i} \leq a_{i+1-} / r_{i+1}$ for every $i<k$. Let $f: X \rightarrow Y$ be a double covering with $X$ smooth curve of genus $g$ and $Y$ smooth curve of genus $q$. If $q \geq 2$ assume $a_{i+} / r_{i}+4 q+3<a_{i+1-} / r_{i+1}$ for every $i<k$ and that $Y$ is bielliptic. Then there is a stable bundle $E$ on $X$ with a filtration $\{E(i)\}_{0 \leq i \leq k}$ with numerical type $\left(k ; a_{1}, r_{1}, \ldots, a_{k}, r_{k}\right)$.

We state explicitely the case $q=2$ of Theorem 0.1 . 
Corollary 0.2. Fix integers $g, k, a_{i}, 1 \leq i \leq k, r_{i}, 1 \leq i \leq k$. Assume $r_{i}>0$ for every $i \geq 1, k \geq 2,2 g>k+6$ and $\left(a_{i+}+11\right) / r_{i}<a_{i+1-} / r_{i+1}$ for every $i<k$. Let $f: X \rightarrow Y$ be a double covering with $X$ smooth curve of genus $g$ and $Y$ bielliptic curve of genus 2. Then there is a stable bundle $E$ on $X$ with a filtration $\{E(i)\}_{0 \leq i \leq k}$ with numerical type $\left(k ; a_{1}, r_{1}, \ldots, a_{k}, r_{k}\right)$.

By Lemma 2.7 proved in Section 2 and Corollary 0.2 we have the following result.

Theorem 0.3. Fix integers $g, k, a_{i}, 1 \leq i \leq k, r_{i}, 1 \leq i \leq k$. Assume $r_{i}>0$ for every $i \geq 1, k \geq 2,2 g>k+6$ and $\left(a_{i+}+11\right) / r_{i}<a_{i+1-} / r_{i+1}$ for every $i<k$. Let $X$ be a general smooth curve of genus $g$. Then there is a stable bundle $E$ on $X$ with a filtration $\{E(i)\}_{0 \leq i \leq k}$ with numerical type $\left(k ; a_{1}, r_{1}, \ldots, a_{k}, r_{k}\right)$.

The proof of 0.3 shows the power of the "double covering tricks" used to prove Theorem 0.1 and the power of a statement like 0.1 for double coverings. We believe that for this type of problems and for the Brill-Noether theory there is a deep difference between double coverings and curves with general moduli. Sometimes (as here and in $[\mathbf{B R}]$ for a weak form of Lange's conjecture) a result proven for double coverings implies the corresponding result for the general curves with the same genus. Sometimes, the theorem for curves with general moduli may be used to obtain the corresponding statement substituting the words "stable bundle" with "semistable bundle" on all smooth curve with the same genus (see e.g. [T], Proof of Th. 0.2). Sometimes, the corresponding result is trivially false for curves with general moduli. In Section 1 we fix the notations used in this paper and we consider the case $g=1$. These results on elliptic curves will be use to prove the case $q=1$ of Theorem 0.1 .

This research was partially supported by MURST and GNSAGA of CNR (Italy).

\section{Elliptic curves.}

We work over an algebraically closed field $\mathbf{K}$ with $\operatorname{char}(\mathbf{K})=0$. For a vector bundle $A$ on a smooth curve, let $\mu(A):=\operatorname{deg}(A) / \operatorname{rank}(A)$ be its slope; if $A$ is semistable set $\mu_{+}(A)=\mu_{-}(A):=\mu(A)$; if $A$ is not semistable, let $\mu_{+}(A)$ (resp. $\left.\mu_{-}(A)\right)$ be the maximal (resp. minimal) slope of a graded subquotient of the Harder-Narasimhan filtration of $A$. If $X$ is a smooth curve of genus $\geq 2$ and $r, d$ are integers with $r \geq 1 M(X ; r, d)$ will denote the variety of all stable vector bundles on $X$ with rank $r$ and degree $d$. We will meet often the following situation. Fix an integer $r \geq 2$. Let $Z$ be a smooth projective curve and $M_{i} \in \operatorname{Pic}(Z), 1 \leq i \leq r$. Set $m_{i}:=\operatorname{deg}\left(M_{i}\right)$. Set $E(0):=\{0\}$ and 
$E(1):=M_{1}$. For $1 \leq i \leq r$ we have an exact sequence

$$
0 \rightarrow E(i-1) \rightarrow E(i) \rightarrow M_{i} \rightarrow 0 .
$$

Hence each $E(i), 1 \leq i \leq r$, is a rank $i$ vector bundle on $Z$ with $\operatorname{deg}(E(i))=$ $\sum_{1 \leq j \leq i} m_{j}$. We will call numerical datum an ordered set of integers $\left[r ; m_{1}, \ldots, m_{r}\right]$ with $r \geq 2$ and we will say that the filtered bundle $\{E(i)\}_{0 \leq i \leq r}$ with $E(i) / E(i-1) \cong M_{i}$ has numerical datum $\left[r ; m_{1}, \ldots, m_{r}\right]$.

Let $E$ be a rank $r$ vector bundle on the smooth curve $Z$ and $P \in Z$. Let $\mathbf{K}_{P}$ be the skyscraper sheaf on $Z$ of length 1 supported by $P$. Fix surjections $u: E \rightarrow \mathbf{K}_{P}$ and $v: E(P) \rightarrow \mathbf{K}_{P}^{\oplus(r-1)}$. The sheaves $\operatorname{Ker}(u)$ and $\operatorname{Ker}(v)$ are locally free of rank $r$. We have $\operatorname{det}(\operatorname{Ker}(u)) \cong \operatorname{det}(E)(-P)$ and $\operatorname{det}(\operatorname{Ker}(v)) \cong \operatorname{det}(E)(P)$. In particular we have $\operatorname{deg}(\operatorname{Ker}(u))=\operatorname{deg}(E)-1$ and $\operatorname{deg}(\operatorname{Ker}(v))=\operatorname{deg}(E)+1$. We will say that $\operatorname{Ker}(u)$ is obtained from $E$ by an elementary transformation supported by $P$ and that $\operatorname{Ker}(v)$ is obtained from $E$ by a +elementary transformation supported by $P$. Note that $\operatorname{Ker}(v)^{*}$ is obtained from $E^{*}$ by an elementary transformation supported by $P$ and that $\operatorname{Ker}(u)^{*}$ is obtained from $E^{*}$ by a +elementary transformation supported by $P$. Furthermore, $E$ is obtained from $\operatorname{Ker}(u)(\operatorname{resp} . \operatorname{Ker}(v))$ by a +elementary (resp. an elementary) transformation supported by $P$.

In this section we will consider the case of a smooth elliptic curve $Y$. The results contained in this section will be used to prove the case $q=1$ of 0.1 . The following Lemma was proved in $[\mathbf{B R}]$.

Lemma 1.1 ([BR, Prop. 1.4]). Fix integers $a, b, u, v$ with $1 \leq u<v$ and $a / u<b / v$. Let $Y$ be an elliptic curve and $A, B$ polystable vector bundles on $Y$ with $\operatorname{deg}(A)=a, \operatorname{rank}(A)=u, \operatorname{deg}(B)=b$ and $\operatorname{rank}(B)=v$. Assume that no two direct factors of $A$ are isomorphic and that the same is true for $B$. Then there exists an injection $j: A \rightarrow B$ with $\operatorname{Coker}(j)$ locally free.

Theorem 1.2. Let $Y$ be an elliptic curve. Fix integers $r \geq 2, m_{1}, \ldots, m_{r}$, such that for all integers $i$ with $1 \leq i<r$ we have $\left(\Sigma_{1 \leq j \leq i} m_{i}\right) / i<m_{i}+1$. Then there is a polystable bundle $E=E(r)$ on $Y$ such that $E$ has a filtration $\{E(i)\}_{0 \leq i \leq r}$ by polystable subbundles with $\operatorname{rank}(E(i))=i$ for every $i$ and $E(i+1) / E(i) \in \operatorname{Pic}^{m_{i}+1}(Y)$, i.e. a filtration determined by exact sequences (1) with numerical datum $\left[r ; m_{1}, \ldots, m_{r}\right]$.

Proof. Fix an integer $i$ with $1 \leq i \leq r$. Let $E(i)$ be a polystable bundle with $\operatorname{rank}(E(i))=i, \operatorname{deg}(E(i))=\Sigma_{1 \leq j \leq i} m_{i}$ and such that no two of the indecomposable factors of $E(i)$ are isomorphic. By assumption we have $\mu(E(i))<\mu(E(i+1))$ for every $i<r$. By Lemma 1.1 there is an inclusion $j: E(i) \rightarrow E(i+1)$ with $\operatorname{Coker}(j)$ locally free, i.e. with $E(i+1) / j(E(i)) \in$ $\operatorname{Pic}^{m_{i+1}}(Y)$. Hence we conclude. 
Lemma 1.3. Let $A$ and $B$ be semistable vector bundles on an elliptic curve $Y$. Assume $\operatorname{rank}(A)>\operatorname{rank}(B)$ and $\mu(A)<\mu(B)-1$. Then there is a surjection $t: A \rightarrow B$.

Proof. Set $u:=\operatorname{rank}(A)$ and $v:=\operatorname{rank}(B)$. Set $H:=H^{0}(Y, \operatorname{Hom}(A, B))$ and for every $P \in X$ set $H(-P):=H^{0}(Y, \operatorname{Hom}(A, B)(-P))$. Since $\operatorname{char}(\mathbf{K})=0$ the bundle $\operatorname{Hom}(A, B)$ is semistable of slope $\mu(B)-\mu(A)>1$. Hence for every $P \in Y$ we have $H^{1}(Y, \operatorname{Hom}(A, B)(-P))=H^{1}(Y, \operatorname{Hom}(A, B))=0$. Thus $\operatorname{dim}(H(-P))=\operatorname{dim}(H)-u v \geq 0$ by Riemann-Roch. Note that the origin $\{0\}$ of the fiber $B_{P}$ of $B$ over $P \in Y$ is the intersection of $v$ hyperplanes of $B_{P}$. This implies that for every $P \in Y$ and for every hyperplane $M$ of the vector space $B_{P}$ the set $H\{P, M\}:=\left\{f \in H: f\left(A_{P}\right) \subseteq M\right\}$ has codimension $\geq u$ in $H$. Since $\mathbf{P}\left(B_{P}\right)=\mathbf{P}^{v-1}$, this implies that the set $H\{P\}:=\{f \in H$ : $\left.f\left(A_{P}\right) \neq B_{P}\right\}$ is an algebraic subset of $H$ with codimension $\geq u-v+1 \geq$ 2. Since $\operatorname{dim}(Y)=1$, this implies that a general $f \in H$ is surjective, as wanted.

The proof of Lemma 1.3 just given shows (just changing the notations) the following more general result.

Lemma 1.4. Let $A$ and $B$ be vector bundles on an elliptic curve $Y$. Assume $\operatorname{rank}(A)>\operatorname{rank}(B)$ and $\mu_{+}(A)<\mu_{-}(B)-1$. Then there is a surjection $t: A \rightarrow B$.

As a particular case of Lemma 1.4 we have the following partial generalization of Theorem 1.2.

Proposition 1.5. Fix an elliptic curve $Y$ and a numerical datum $\left[r ; m_{1}, \ldots, m_{r}\right]$. Let $E$ be a semistable vector bundle on $Y$ with $\operatorname{rank}(E)=r$ and $\operatorname{deg}(E)=\Sigma_{1 \leq j \leq r} m_{r}$. Assume that $m_{r} \geq m_{i}+2$ for every integer $i<r$. Fix $M_{i} \in \operatorname{Pic}^{m_{i}}(Y), 1 \leq i \leq r$, such that $\otimes_{1 \leq i \leq r} M_{i} \cong \operatorname{det}(E)$. Set $E(r):=E$ and for $1 \leq i<r$ set $E(i):=\oplus_{1 \leq j \leq i} M_{i}$. Then there exists an inclusion $m: E(r-1) \rightarrow E$ with $M_{r} \cong \operatorname{Coker}(m)$. In particular $E$ has a filtration $\{E(i)\}_{0 \leq i \leq r}$ with $E(i-1), E(i)$ and $M_{i}$ fitting in the exact sequences (1).

Now we may generalize Proposition 1.5 in the following way.

Theorem 1.6. Fix an elliptic curve $Y$ and a numerical datum $\left[r ; m_{1}, \ldots\right.$, $\left.m_{r}\right]$. Let $E$ be a semistable vector bundle on $Y$ with $\operatorname{rank}(E)=r$ and $\operatorname{deg}(E)=\Sigma_{1 \leq j \leq r} m_{r}$. Assume the existence of an integer $k \geq 1$ and of integers $0:=i(0)<i(1)<\cdots<i(k):=r$ such that for every integer $t$ with $1 \leq$ $t \leq k$ we have $m_{i(t)} \geq 2+\max \left\{m_{j}\right\}_{i(t-1)<j<i(t)}$ and such that if $t<k$ we have $\left(\Sigma_{i(t-1)<j \leq i(t)} m_{j}\right) /(i(t)-i(t-1))<1+\left(\Sigma_{i(t)<j \leq i(t+1)} m_{j}\right) /(i(t+1)-i(t))$. Then 
there exists a semistable vector bundle $E$ on $X$ with a filtration $\{E(i)\}_{0 \leq i \leq r}$ with numerical datum $\left[r ; m_{1}, \ldots, m_{r}\right]$ and such that for all integers $t$ with $1 \leq t \leq k$, the bundle $E(i(t)) / E(i(t-1))$ is semistable.

Proof. If $k=1$, this is Proposition 1.5. Assume $k>1$. For every integer $t$ with $1 \leq t \leq k$ we apply 1.5 to the numerical datum $[i(t)-i(t-$ $\left.1), m_{i}(t-1)+1, \ldots, m_{i}(t)\right]$. Since $m_{i}(t) \geq 2+\max \left\{m_{j}\right\}_{i(t-1)<j<i(t)}$ we obtain a semistable bundle $F(t)$ with $\operatorname{rank}(F(t))=i(t)-i(t-1)$ and $\operatorname{deg}(F(t))=$ $\Sigma_{i(t-1)<j \leq i(t)} m_{j}$. Set $E(i(1)):=F(1)$ and take the corresponding filtration. Since $\mu(F(2))>1+\mu(F(1))$ by Lemma 1.3 there is a semistable bundle $E(i(2))$ with $E(i(1))$ subbundle of $E(i(2))$ and with $E(i(2)) / E(i(1)) \cong F(2)$. If $k=2$ by Lemma 1.3 we may take $E(i(2))$ as $E$. If $k>2$ we continue in the same way.

Remark 1.7. Here we analyze the cases with $r=3$ not covered by Theorem 1.2. First we assume $m_{2}>m_{1}$. If there is any chance to find a semistable bundle with numerical datum $\left(3 ; m_{1}, m_{2}, m_{3}\right)$ we need (and will assume) $3 m_{1} \leq m_{1}+m_{2}+m_{3}$ (i.e. $\left.-m_{3}-m_{2} \leq-2 m_{1}\right)$ and $m_{1}+$ $m_{2} \leq 2 m_{3}$. Since the dual of a stable bundle is stable, we will consider the datum $\left(3 ;-m_{3},-m_{2},-m_{1}\right)$. Since the numerical datum $\left(3 ; m_{1}, m_{2}, m_{3}\right)$ is not covered by 1.2 we have $-m_{3}<-m_{2}$. If we have $-m_{3}-m_{2}<-2 m_{1}$ we may apply Theorem 1.2 to this datum. Assume $-m_{3}-m_{2}=-2 m_{1}$. Let $B$ be a polystable bundle with a filtration of numerical type $\left(2 ;-m_{3},-m_{2}\right)$. Take as polystable bundle $B \oplus R$ with $R \in \operatorname{Pic}^{-m_{1}}(Y)$. Now we assume $m_{1}=m_{2}$. We apply Lemma 1.1 taking $A:=M_{1} \oplus M_{2}$ with $M_{1}$ and $M_{2}$ not isomorphic. If $m_{1}<m_{2}=m_{3}$ we may apply this discussion to the dual numerical datum $\left(3 ;-m_{3},-m_{2},-m_{1}\right)$. If $m_{1}=m_{2}=m_{3}$ the construction of a polystable bundle with numerical datum $\left(3 ; m_{1}, m_{2}, m_{3}\right)$ is trivial.

\section{A few lemmas.}

In this section we collect several lemmas.

Lemma 2.1. Fix an integer $g \geq 2$, a numerical datum $\left[r ; m_{1}, \ldots, m_{r}\right]$ and a smooth genus $g$ curve $Z$. Assume the existence of a stable (resp. semistable) bundle $E$ on $Z$ associated to the numerical datum $\left[r ; m_{1}, \ldots, m_{r}\right]$. Assume the existence of line bundles $M_{i} \in \operatorname{Pic}^{m_{i}}(Z), 1 \leq i \leq r$, such that $h^{0}\left(Z, \operatorname{Hom}\left(M_{i}, M_{j}\right)\right)=0$ for all integers $i, j$ with $1 \leq j<i \leq r$. Then for a general curve $X$ of genus $g$ there is a stable (resp. semistable) bundle associated to the numerical datum $\left[r ; m_{1}, \ldots, m_{r}\right]$.

Proof. Let $\{E(i)\}_{0 \leq i \leq r}$ be the filtration of $E$ with $M_{i}, 1 \leq i \leq r$, as graded subquotients. The cohomological assumption gives inductively that for all 
integers $i$ with $1 \leq i<r$ we have $\operatorname{dim}\left(\operatorname{Ext}^{1}\left(M_{i+1}, E(i)\right)\right)=-\chi\left(\operatorname{Hom}\left(M_{i+1}\right.\right.$, $E(i)))=-\Sigma_{1 \leq j \leq i} \chi\left(\operatorname{Hom}\left(M_{i+1}, M_{j}\right)\right)$, i.e. the minimal possible value. Note that on a finite covering $M$ of $M_{g, 1}$ there are the relative Picard varieties Pic $^{t}$ for all integers $t$. Since every vector bundle on an integral variety has integral total space and the associated relative Ext ${ }^{1}$-functor is a vector bundle over a Zariski dense open subset of $M$ by semicontinuity, we conclude by the openness of stability (resp. semistability) and the theory of relative Ext ([BPS] or $[\mathbf{L} 1])$.

Remark 2.2. The cohomological assumption of Lemma 2.1 is always satisfied if the numerical datum $\left[r ; m_{1}, \ldots, m_{r}\right]$ is strictly increasing, i.e. $m_{j}<m_{i}$ for all $i, j$ with $1 \leq j<i \leq r$, or if $\left[r ; m_{1}, \ldots, m_{r}\right]$ is weakly increasing, i.e. $m_{j} \leq m_{i}$ for all $i, j$ with $j<i$, and for no pair $(i, j)$ with $i \neq j M_{i}$ and $M_{j}$ are isomorphic.

The proof of Lemma 2.1 gives the following result.

Lemma 2.3. Fix an integer $g \geq 2$, a numerical type $\left(k ; a_{1}, r_{1}, \ldots, a_{k}, r_{k}\right)$, and a smooth genus $g$ curve $Z$. Assume the existence of a stable (resp. semistable) bundle $E$ on $Z$ with a filtration $\left\{E_{i}\right\}_{0 \leq i \leq k}$ with numerical type $\left(k ; a_{1}, r_{1}, \ldots, a_{k}, r_{k}\right)$ and such that $H^{0}\left(Z, \operatorname{Hom}\left(E_{i} / E_{i-1}, E_{j} / E_{j-1}\right)\right)=0$ for all integers $i, j$ with $0<j<i \leq k$. Then on a general smooth curve there is a stable (resp. semistable) bundle $E^{\prime}$ with a filtration of type $\left(k ; a_{1}, r_{1}, \ldots\right.$, $\left.a_{k}, r_{k}\right)$. Furthermore, if some of the bundles $E_{i} / E_{i-1}$ are stable or semistable or simple, we may find $E^{\prime}$ such that the corresponding bundles $E_{i}^{\prime} / E_{i-1}^{\prime}$ have the same properties.

The proof of Lemma 1.3 gives verbatim the following results.

Lemma 2.4. Let $Z$ be a smooth curve of genus $q \geq 2$. Fix vector bundles $A, B, D$ with $\mu_{+}(A)+2 q-1<\mu_{-}(B), \operatorname{rank}(A)<\operatorname{rank}(B), \operatorname{rank}(B)>$ $\operatorname{rank}(D)$ and $\mu_{+}(B)+2 q-1<\mu_{-}(D)$. Then there is an embedding $j: A \rightarrow B$ with $\operatorname{Coker}(j)$ locally free and a surjection $B \rightarrow D$.

Lemma 2.5. Let $Z$ be a smooth curve of genus $q \geq 2$. Fix stable vector bundles $A, B, D$ with $\mu(A)+2 q-1<\mu(B), \operatorname{rank}(A)<\operatorname{rank}(B), \operatorname{rank}(B)>$ $\operatorname{rank}(D)$ and $\mu(B)+2 q-1<\mu(D)$. Then there is an embedding $j: A \rightarrow B$ with $\operatorname{Coker}(j)$ locally free and a surjection $B \rightarrow D$.

We will use often the following notations. We have integers $a, b, r, s, q$ with $r>0, s>0, q \geq 2$. We consider an exact sequence

$$
0 \rightarrow H \rightarrow E \rightarrow Q \rightarrow 0
$$


of vector bundles on a smooth genus $q$ curve called $Z$ (or sometimes $X$ ) with $\operatorname{rank}(H)=r, \operatorname{rank}(Q)=s, \operatorname{deg}(H)=a, \operatorname{deg}(Q)=b$. Usually we will have $a / r<b / s$ and we will look at exact sequences (2) with $H, E$ and $Q$ stable.

Lemma 2.6. Let $Z$ be a smooth curve of genus $q \geq 2$. Fix integers $a, b, r, s$ with $r>0, s>0, a / r+4 q-2<2 q-1+(a+b) /(r+s)<b / s$. Fix $Q \in M(Z ; s, b)$. Then there is an exact sequence $(2)$ in which $E$ is a general element of $M(Z ; r+s, a+b)$ and $H$ is a general element of $M(Z ; r, a)$.

Proof. By Lemma 2.4 for every $E \in M(Z ; r+s, a+b)$ there is a surjection $t: E \rightarrow Q$. We fix any such surjection $t$ and set $H:=\operatorname{Ker}(t)$. Since $H$ is a flat limit of a flat family of stable bundles and $H^{1}(Z, \operatorname{Hom}(E, Q))=0$, it is sufficient to check that varying the bundle $E$ and the surjection $t$ we obtain as $\operatorname{Ker}(t)$ all bundles in a neighborhood of $H$ in its semiuniversal deformation space. By deformation theory $H^{1}(Z, \operatorname{Hom}(H, H))$ is the tangent space to this deformation space and $H^{1}(Z, \operatorname{Hom}(E, E))$ is the tangent space to the deformation space of $E$ which is smooth (i.e. every infinitesimal deformation lifts to a local deformation) of dimension $h^{1}(Z, \operatorname{Hom}(E, E))=$ $-\chi(\operatorname{Hom}(E, E))+1$ by the stability of $E$. Applying the functors $\operatorname{Hom}(-, H)$ and $\operatorname{Hom}(E,-)$ to $(2)$ we obtain the exact sequences

$$
H^{1}(Z, \operatorname{Hom}(Q, H)) \rightarrow H^{1}(Z, \operatorname{Hom}(E, H)) \rightarrow H^{1}(Z, \operatorname{Hom}(H, H)) \rightarrow 0
$$

$$
H^{0}(Z, \operatorname{Hom}(E, Q)) \rightarrow H^{1}(Z, \operatorname{Hom}(E, H)) \rightarrow H^{1}(Z, \operatorname{Hom}(E, E)) .
$$

Hence we conclude.

The proof of Lemma 2.1 gives the following result.

Lemma 2.7. Fix an integer $g \geq 2$, a numerical type $\left(k ; a_{1}, r_{1}, \ldots, a_{k}, r_{k}\right)$ and a smooth genus $g$ curve $Z$. Assume the existence of a stable (resp. semistable) bundle $E$ on $Z$ with a filtration $\left\{E_{i}\right\}_{0 \leq i \leq k}$ with numerical type $\left(k ; a_{1}, r_{1}, \ldots, a_{k}, r_{k}\right)$ such that $h^{0}\left(Z, \operatorname{Hom}\left(E_{i} / E_{i-1}, E_{i-1}\right)\right)=0$ for every integer $i$ with $2 \leq i \leq r$. Then for a general curve $X$ of genus $g$ there is a stable (resp. semistable) bundle with a filtration of numerical type $\left(k ; a_{1}, r_{1}, \ldots, a_{k}, r_{k}\right)$.

\section{Proofs of $0.1,0.2$ and 0.3 .}

In this section we prove Theorem 0.1 and hence Corollary 0.2 and (by Lemma 2.7) Theorem 0.3. Then we give a related result (Proposition 3.1). 
Proof of Theorem 0.1. Let $\sigma \in \operatorname{Aut}(X)$ be the involution determined by $f$ and $B(f)$ the ramification locus of $f$. By assumption we have $2 g+2>4 q$. We have $\operatorname{card}(B(f))=2 g+2-4 q$ by Riemann-Hurwitz. Since $B(f) \neq \varnothing$, for every stable bundle $M$ on $Y$ the bundle $f^{*}(M)$ is stable (see e.g. [BBR], Lemma 2.2). The proof will be divided into two steps. In the first step we will prove the case $q \geq 2$, while in the second step we will consider the case $q=1$.

Step 1. Assume $q \geq 2$. Set $I:=\left\{i \in\{1, \ldots, k\}: a_{i}\right.$ is odd $\}$ and $t:=\operatorname{card}(I)$. Hence $0 \leq t \leq k$. Fix $t$ general points of $Y$, say $f(P(x)), 1 \leq x \leq t$, with $P(x) \in X, 1 \leq x \leq t$, and $\operatorname{card}\{P(x), \sigma(P(x)), 1 \leq x \leq t\}=2 t$. Set $b_{i}^{\prime}:=$ $b_{i}^{\prime \prime}:=a_{i} / 2$ if $a_{i}$ is even and $b_{i}^{\prime}:=b_{i}^{\prime \prime}-1:=\left[a_{i} / 2\right]$ if $a_{i}$ is odd. Let $F^{\prime}\left(\right.$ resp. $\left.F^{\prime \prime}\right)$ be a stable bundle on $Y$ with a filtration $\left\{F^{\prime}(i)\right\}_{0 \leq i \leq k}$ (resp. $\left.\left\{F^{\prime \prime}(i)\right\}_{0 \leq i \leq k}\right)$ of type $\left(k ; b_{1}^{\prime}, r_{1}, \ldots, b_{k}^{\prime}, r_{k}\right)$ (resp. $\left.\left(k ; b_{1}^{\prime \prime}, r_{1}, \ldots, b_{k}^{\prime \prime}, r_{k}\right)\right)$, with $F^{\prime}$ subsheaf of $F^{\prime \prime}$ and such that $F^{\prime \prime}(i) / F^{\prime \prime}(i-1)$ is obtained from $F^{\prime}(i) / F^{\prime}(i-1)$ by one +elementary transformation if $a_{i}$ is odd and $F^{\prime \prime}(i) / F^{\prime \prime}(i-1)=F^{\prime}(i) / F^{\prime}(i-1)$ if $a_{i}$ is even, each +elementary transformation supported by a different point of $\{f(P(x))\}_{1 \leq x \leq t}$; the existence of $F$ and $F^{\prime}$ follows taking +elementary transformations of suitable bundle $\pi^{*}\left(A^{\prime}\right)$ and $\pi^{*}\left(A^{\prime \prime}\right)$ with $\pi: Y \rightarrow Z$ double covering, $Z$ elliptic curve and $A^{\prime}, A^{\prime \prime}$ polystable vector bundles on $Z$; for more details, see a similar situation in the next few lines. Set $E^{\prime}:=f^{*}\left(F^{\prime}\right)$ and $E^{\prime \prime}:=f^{*}\left(F^{\prime \prime}\right)$. Let $E$ be the unique bundle containing $E^{\prime}$, contained in $E^{\prime \prime}$ and such that $E$ is obtained from $E^{\prime}$ making $t+$ elementary transformations supported by the points $P(x), 1 \leq x \leq t$. Note that $E$ is obtained from $E^{\prime \prime}$ making $t$ elementary transformations supported by the points $\sigma(P(x))$, $1 \leq x \leq t$. Note that $E^{\prime \prime}$ is the minimal bundle containing both $E$ and $\sigma(E)$. By construction $E$ has a filtration $\{E(i)\}_{0 \leq i \leq k}$ of type $\left(k ; a_{1}, r_{1}, \ldots, a_{k}, r_{k}\right)$. It is sufficient to show the stability of $E$. In order to obtain a contradiction we assume the existence of a saturated subbundle $A$ of $E$ with $\mu(A) \geq \mu(E)$. Taking $\operatorname{rank}(A)$ minimal, we may assume $A$ stable. We have $A \neq \sigma(A)$ because $A \cap \sigma(A)$ is contained in $E^{\prime}$ and $E^{\prime}$ is stable with $\mu\left(E^{\prime}\right) \leq \mu(E)$. By [BR], Step 4 of the proof of Th. 0.1, there are bundles $B, U$ on $Y, B$ subsheaf of $F^{\prime \prime}, U$ saturated subsheaf of $B$, with $A \cap \sigma(A)=f^{*}(U)$ and $A+\sigma(A)=$ $f^{*}(B)$. Since $E^{\prime \prime}$ comes from $Y$, for every $Q \in B(f) \subset Y$ the involution $\sigma$ acts as the identity over the fiber $E_{Q^{\prime}}^{\prime \prime}$ over the point $Q^{\prime}:=f^{-1}(Q)_{\text {red }} \in X$. Hence the inclusion of the subsheaf $A+\sigma(A)$ into $E^{\prime \prime}$ has $A_{Q^{\prime}}$ as image into $E_{Q^{\prime}}^{\prime \prime}$, i.e. calling $K$ the saturation of $A+\sigma(A)$ into $E^{\prime \prime}, K /(A+\sigma(A))$ has length $\geq \operatorname{rank}(A+\sigma(A))-\operatorname{rank}(A)$ at each of the $\operatorname{card}(B(f))$ ramification points of $f$. Since $E^{\prime \prime}$ is stable and $A+\sigma(A)$ is a quotient of the polystable bundle $A \oplus \sigma(A)$ we have $\mu(A+\sigma(A)) \geq \mu(A)$ and $\mu\left(E^{\prime \prime}\right)=\mu(E)+t / r \geq$ $\mu(K) \geq \operatorname{card}(B(f)) \cdot(\operatorname{rank}(A+\sigma(A))-\operatorname{rank}(A))+\mu(A)$, with equality only if $A+\sigma(A) \cong A \oplus \sigma(A)$ and $K=E^{\prime \prime}$ (and hence $\operatorname{rank}(A)=r / 2$ ). In all 
cases if $2 g+2>k+4 q$ we obtain a contradiction.

Step 2. Here we assume $q=1$. If $a_{i}$ is even set $b_{i}^{\prime}:=a_{i} / 2-2$ and $b_{i}^{\prime}:=a_{i} / 2$; if $a_{i}$ is odd set $b_{i}^{\prime}:=\left[a_{i} / 2\right]$ and $b_{i}^{\prime \prime}:=\left[a_{i} / 2\right]+1$. Let $t$ be the number of indices $i$ with $a_{i}$ odd. Choose $2 k-t$ general points of $Y$, say $f(P(x))$, $1 \leq x \leq 2 k-t$, with $P(x) \in X$ and $\{P(1), \ldots, P(2 k-t)\}$ general. Let $F^{\prime}$ (resp. $F^{\prime \prime}$ ) be polystable bundles on $Y$ with a filtration $\left\{F^{\prime}(i)\right\}_{0 \leq i \leq k}$ (resp. $\left.\left\{F^{\prime \prime}(i)\right\}_{0 \leq i \leq k}\right)$ of type $\left(k ; b_{1}^{\prime}, r_{1}, \ldots, b_{k}^{\prime}, r_{k}\right)\left(\operatorname{resp} .\left(k ; b_{1}^{\prime \prime}, r_{1}, \ldots, b_{k}^{\prime \prime}, r_{k}\right)\right)$, with $F^{\prime}$ subsheaf of $F^{\prime \prime}$ and such that $F^{\prime \prime}(i) / F^{\prime \prime}(i-1)$ is obtained from $F^{\prime}(i) / F^{\prime}(i-$ 1 ) by one +elementary transformation if $a_{i}$ is odd and by two +elementary transformations if $a_{i}$ is even, each +elementary transformation supported by a different point of $\{f(P(x))\}_{1 \leq x \leq 2 k-t}$. Such bundles $F^{\prime}$ and $F^{\prime \prime}$ exists by Lemma 1.1. Note that here we could use also the more elementary Lemma 1.3. As in Step 1 we obtain a contradiction if $2 g-2:=\operatorname{card}(B(f))>2 k-t$. Since $t \geq 0$, here it is sufficient to assume $g \geq k$.

Proposition 3.1. Fix integers $g, r, m_{i}, 1 \leq i \leq r$, with $g \geq 2$. Let $u$ be an integer $i \leq r$ with $m_{i}$ maximal. Set $\Delta\left(\left\{m_{i}\right\}\right):=\Sigma_{1 \leq i \leq r\left(m_{u}-m_{i}\right)}$. Fix any integer $t \geq 1+\Delta\left(\left\{m_{i}\right\}\right)$. Let $X$ be a smooth genus $g$ curve. Set $m_{i}^{\prime}:=m_{u}$ for $i<r, m_{r}^{\prime}:=m_{u}+t-\Delta\left(\left\{m_{i}\right\}\right)$. Then a general rank $r$ vector bundle on $X$ with a filtration of type $\left\{m_{i}^{\prime}\right\}_{1 \leq i \leq r}$ is stable.

Proof. Choose general bundles $M_{i} \in \operatorname{Pic}^{m_{i}}(X), 1 \leq i \leq r$, and general bundles $M_{i}^{\prime} \in \operatorname{Pic}^{m_{u}}(X), 1 \leq i \leq r$, with $h^{0}\left(X, M_{i}^{\prime} \otimes M_{i}^{*}\right) \neq 0$ for all $i$. Set $F:=\oplus_{1 \leq i \leq r} M_{i}, F^{\prime}:=\oplus_{1 \leq i \leq r} M_{i}^{\prime}, G:=\oplus_{1 \leq i<r} M_{i}$ and $F^{\prime \prime}:=G \oplus M_{r}^{\prime}$. Since $M_{i}^{\prime}$ is obtained from $M_{i}$ adding an effective divisor of degree $m_{u}-m_{i}$, $F^{\prime}$ is obtained from $F$ making $\Delta\left(\left\{m_{i}\right\}\right)$ +elementary transformations. For general $M_{i}$ 's we may assume that no two $M_{i}^{\prime}$ 's are isomorphic. By [B], Prop. 2.3 and 2.7, for every integer $w \geq 1$ a general bundle obtained from $F^{\prime}$ making $w$ +elementary transformations is stable. Take $w:=t-\Delta\left(\left\{m_{i}\right\}\right)$.

The family of bundles obtained in this way is contained in the closure of the family of bundles obtained from $F^{\prime \prime}$ making $t-m_{u}+m_{r}$ general +elementary transformations. Any such bundle, $A$, has $G$ as subbundle and hence it has a filtration of type $m_{1}^{\prime}, \ldots, m_{r}^{\prime}:=\operatorname{deg}(A)-\Sigma_{1 \leq i<r} m_{i}^{\prime}$. Hence we conclude by the openness of stability.

\section{Other results.}

In this section we will use the following notations. Fix a smooth curve $X$ of genus $g \geq 2$, an integer $r \geq 2$ and integers $m_{i}, 1 \leq i \leq r$. Set ext $:=$ $\operatorname{ext}\left(\left\{m_{i}\right\}\right): \Sigma_{1 \leq j<i \leq r}\left(m_{i}-m_{j}+g-1\right)=\Sigma_{1 \leq i \leq r}(2 i-r-1) m_{i}+r(r-1)(g-1) / 2$. Let $E$ be a "general" rank $r$ bundle with a filtration $\{E(i)\}_{0 \leq i \leq r}$ of type 
$\left(r ; m_{1}, 1, \ldots, m_{r}, 1\right)$, i.e. with numerical datum $\left[r ; m_{1}, \ldots, m_{r}\right]$. Set $M_{i}:=$ $E_{i} / E_{i-1} \in \operatorname{Pic}^{m_{i}}(X)$. We assume that $\left(M_{1}, \ldots, M_{r}\right)$ is a general element of $\operatorname{Pic}^{m_{1}}(X) \times \cdots \times \operatorname{Pic}^{m_{r}}(X)$. We always assume $m_{j} \leq m_{i}+g-1$ for all $i, j$ with $j<i$. When this condition is satisfied, for general $\left(M_{1}, \ldots, M_{r}\right) \in$ $\operatorname{Pic}^{m_{1}}(X) \times \cdots \times \operatorname{Pic}^{m_{r}}(X)$ the following condition (Condition (\$)) is satisfied:

$$
\text { Condition }(\$): \quad h^{0}\left(X, \operatorname{Hom}\left(M_{i}, M_{j}\right)\right)=0 \text { if } i>j .
$$

When Condition $(\$)$ is satisfied, for fixed $\left(M_{1}, \ldots, M_{r}\right)$ the set $\mathbf{T}\left(M_{1}, \ldots\right.$, $M_{r}$ ) of all filtered bundles with $\left\{M_{i}\right\}_{1 \leq i \leq r}$ as graded subquotients is a vector space of dimension ext. Varying $\left(M_{1}, \ldots, M_{r}\right) \in \operatorname{Pic}^{m_{1}}(X) \times \cdots \times$ $\operatorname{Pic}^{m_{r}}(X)$ among the $r$-ples satisfying Condition (\$) we obtain an irreducible family, $\mathbf{T}$, of dimension $r g+$ ext of filtered bundles with numerical type $\left(r ; m_{1}, 1, \ldots, m_{r}, 1\right) . E$ will be a general element of $\mathbf{T}$.

Lemma 4.1. Fix $M_{i} \in \operatorname{Pic}^{m_{i}}(X), 1 \leq i \leq r$, and assume Condition (\$). Let $F$ be the general bundle on $X$ with a filtration $\{F(i)\}_{0 \leq i \leq r}$ with $F(i) / F(i-$ $1) \cong M_{i}$. Let $R$ be a rank 1 subbundle of $F$ contained in $F(k), 2 \leq k \leq r$, but not in $F(k-1)$. Then $(k+1) \operatorname{deg}(R) \leq \Sigma_{1 \leq j<k} m_{j}+2 m_{k}-(k-1)(g-1)$.

Proof. By assumption the inclusion $R \rightarrow F(k)$ induces a non-zero map $R \rightarrow M_{k}$ and hence an effective divisor $D$ with $R \cong M_{k}(-D)$. Furthermore, the inclusion $R \rightarrow F(k)$ induces an inclusion $F(k-1) \oplus R \rightarrow F(k)$. Set $a:=\operatorname{deg}(D) \geq 0 . F(k)$ is obtained from $F(k-1) \oplus R$ making a +elementary transformations with supports at points $P_{i}, 1 \leq i \leq a$, (not necessarly different) with $D=\Sigma_{1 \leq i \leq a} P_{i}$. For fixed $R$ and $D$ the set of all such +elementary transformations is parametrized by a vector space of dimension ak. By Condition $(\$)$ we have $\operatorname{dim}\left(\operatorname{Ext}^{1}\left(M_{k}, F(k-1)\right)\right)=(k-1)\left(g-1+m_{k}\right)-\Sigma_{1 \leq i \leq k}-m_{i}$. Note that every inclusion of $F(k-1) \oplus R$ into $F$ induces an extension of $M_{k}$ by $F(k-1)$ with $F$ as middle term. Hence, since $\operatorname{dim}(X)=1$ and the set of all +elementary transformations of a fixed rank $k$ bundle supported at a fixed point $P \in X$ has dimension $k$, we obtain $k a+a \leq \operatorname{dim}\left(\operatorname{Ext}^{1}\left(M_{k}, F(k-1)\right)\right)$, i.e. the lemma.

In particular we obtain the following result.

Corollary 4.2. Fix $M_{i} \in \operatorname{Pic}^{m_{i}}(X), 1 \leq i \leq r$, and assume Condition $(\$)$. Assume that for every integer $k$ with $2 \leq k \leq r$ we have $(k+1) m_{1}>$ $\Sigma_{1 \leq j<k} m_{j}+2 m k-(k-1)(g-1)$. Let $F$ be the general bundle on $X$ with a filtration $\{F(i)\}_{0 \leq i \leq r}$ with $F(i) / F(i-1) \cong M_{i}$. Then every rank 1 subbundle of $F$ has degree $\leq m_{1}$ and $M_{1}:=F(1)$ is the unique subbundle of $E$ with degree $m_{1}$.

Corollary 4.3. Fix $M_{i} \in \operatorname{Pic}^{m_{i}}(X), 1 \leq i \leq r$, and assume Condition (\$). Assume that $2 m_{1}>2 m_{2}+1-g$ and that for every integer $k$ with $3 \leq k \leq r$ 
we have $m_{1} \geq m_{k}-\left(2^{k-2}-1\right)(g-1) 2^{2-k}$. Let $F$ be the general bundle on $X$ with a filtration $\{F(i)\}_{0 \leq i \leq r}$ with $F(i) / F(i-1) \cong M_{i}$. Then every rank 1 subbundle of $F$ has degree $\leq m_{1}$ and $M_{1}:=F(1)$ is the unique subbundle of $E$ with degree $m_{1}$.

Proof. By 4.2 we need to check that for all integers $k$ with $2 \leq k \leq r$ we have $(k+1) m_{1}>\Sigma_{1 \leq j<k} m_{j}+2 m_{k}-(k-1)(g-1)$. For $k=2$ we assumed $m_{1}>m_{2}+$ $(1-g) / 2$. For $k=3$ we assumed $4 m_{1}>m_{1}+m_{2}+2 m_{3}+(1-g) / 2+3(1-g) / 2$. And so on, using that $\Sigma_{2 \leq i<k}(1-2-i+1)+2\left(1-2^{-k+1}\right)=k-1$.

Now we may extend Corollary 4.3 to the case of subbundles of rank $b>1$.

Theorem 4.4. Let $X$ be a smooth curve of genus $g \geq 2$. Fix integers $r, b$ with $1 \leq b \leq r$. Fix $M_{i} \in \operatorname{Pic}^{m_{i}}(X), 1 \leq i \leq r$, and assume Condition $(\$)$. Assume $m_{i}>m_{j}-\left(2^{j-i+1}-1\right)(g-1) 2^{i-j-1}$ for all integers $i, j$ with $1 \leq i<j \leq r$. Let $F$ be the general bundle on $X$ with a filtration $\{F(i)\}_{0 \leq i \leq r}$ with $F(i) / F(i-1) \cong M_{i}$. Then every rank $b$ subbundle of $F$ has degree $\leq \Sigma_{1 \leq i \leq b} m_{i}$ and $F(b)$ is the unique subbundle of $F$ with degree $\Sigma_{1 \leq i \leq b} m_{i}$.

Proof. We use double induction on $b$ and $r$, the case $b=1$ being covered by Corollary 4.3. Assume $b \geq 2$ and let $A$ be a rank $b$ saturated subbundle of $F$. Since the case $b=r$ is trivial and $F(r-1)$ is saturated in $F$, we may assume $\operatorname{rank}(A \cap F(r-1))=b-1$ and $b<r$. Hence the image of $A$ into $M_{r}:=F(r) / F(r-1)$ is a subsheaf $M_{r}(-D)$ of $M_{r}$, with $D$ effective divisor on $X$. We have $\operatorname{deg}(A)=\operatorname{deg}(A \cap F(r-1))+\operatorname{deg}\left(M_{r}(-D)\right)$. By the inductive assumption we have $\operatorname{deg}(A \cap F(r-1)) \leq \Sigma_{1 \leq i \leq b-1} m_{i}$. The proof of Corollary 4.3 gives $\operatorname{deg}\left(M_{r}(-D)\right)<m_{1}$. However, our assumptions on the integers $\left\{m_{i}\right\}_{1 \leq i \leq r}$ are stronger than in 4.3 and hence we may apply 4.3 to $F / F(b-1)$ and find $\operatorname{deg}\left(M_{r}(-D)\right)<m_{b}$. Hence we have $\operatorname{deg}(A)<\Sigma_{1 \leq i \leq b} m_{i}$, as wanted.

\section{References}

[B] E. Ballico, Brill-Noether theory for vector bundles on projective curves, Math. Proc. Cambridge Phil. Soc., to appear.

[BBR] E. Ballico, L. Brambila-Paz and B. Russo, Exact sequences of stable vector bundles on projective curves, Math. Nachr, to appear.

[BR] E. Ballico and B. Russo, Exact sequences of semistable vector bundles on algebraic curves, preprint.

[BPS] C. Banica, M. Putinar and G. Schumaker, Variation der globalen Ext in Deformationen kompakter Räume, Math. Ann., 250 (1980), 135-155.

[BL] L. Brambila-Paz and H. Lange, A stratification of the moduli scheme of vector bundles on curves, preprint alg-geom 9708014. 
[L1] H. Lange, Universal families of extensions, J. of Algebra, 83 (1983), 101-112.

[L2] , Zur Klassifikation von Regelmannigfaltigkeiten, Math. Ann., 262 (1984), 447-459.

[T] M. Teixidor i Bigas, On Lange's conjecture, preprint.

Received March 12, 1997 and revised September 17, 1997.

UNIVERSITY OF TRENTO

38050 Povo (TN) ITALY

E-mail address: ballico@science.unitn.it 